\title{
Matriz de análisis competencial, herramienta para el desarrollo de habilidades directivas
}

\section{Análisis competencial's Matrix, tool for the development of management skills}

\author{
Adriel Malvarez Cuello. ${ }^{1}$ Daniel Crispin Rodriguez. ${ }^{2}$ \& Celia Amy Espinosa \\ Gonzalez. ${ }^{3}$
}

\section{DOI: https://doi.org/10.33262/visionariodigital.v4i3.1271}

\begin{abstract}
.
To maintain a correct administration that allows to reach satisfactory results, is a challenge to which fight the companies daily. Among the keys to reach the goals of the company, the acting of their managers is one of the most important. To achieve an effective work, the managers should possess and to apply the abilities that allow him to guide their workers on the wanted road. In the national environment, it is very common to find people occupying positions for those that are not qualified and in general sense, the companies don't pay the due attention to the development of the management skills of their squares. For the diagnose the state of this skills exist diverse technical, which can be applied. For the development of this investigation, it was chosen to the restaurant Don Cangrejo to apply one of these tools and it was pursued as general objective to determine the influence of the behavior of the management skills of the squares of the entity in the development of the administration of the installation. To fulfill this objective a survey it was applied the directive of the local and the results were analyzed leaning on in the methodology used by the Hay Group in the application of the Interview of Critical Incidents. With the results hurtled by the survey, a strategy was proposed and a plan of actions was elaborated for the improvement of the administration in the installation.
\end{abstract}

Keywords: Management Skills, Skills Diagnostic, Strategy.

\section{Resumen.}

\footnotetext{
1 Facultad de Turismo-Universidad de La Habana, Cuba, email adriel_m@estudiantes.ftur.uh.cu, adrielmalva2017@gmail.com

${ }^{2}$ Facultad de Turismo-Universidad de La Habana, Cuba, email, danicr97@yahoo.com

${ }^{3}$ Facultad de Comunicación-Universidad de La Habana, Cuba, email adrielmalva2017@gmail.com
} 
Mantener una correcta gestión, que permita alcanzar resultados satisfactorios, es un reto al que se enfrentan diariamente las empresas. Entre las claves para alcanzar las metas de la empresa, el desempeño de sus directivos figura como una de las más importantes. Para lograr una labor eficaz, los directivos deben poseer y aplicar las habilidades que le permitan guiar a sus trabajadores sobre el camino deseado. En el ámbito nacional es muy común encontrar personas ocupando cargos para los que no están capacitados y en sentido general, las empresas no prestan la debida atención al desarrollo de las habilidades directivas de sus cuadros. Para diagnosticar el estado de dichas habilidades existen diversas técnicas, las cuales pueden ser aplicadas. Para el desarrollo de esta investigación se escogió al restaurante Don Cangrejo para aplicar una de estas herramientas y se persiguió como objetivo general determinar la influencia del comportamiento de las habilidades directivas de los cuadros de la entidad en el desarrollo de la gestión de la instalación. Para cumplir con este objetivo se aplicó una encuesta a los directivos del local y se analizaron los resultados apoyándose en la metodología empleada por el Hay Group en la aplicación de la Entrevista de Incidentes Críticos. Con los resultados arrojados por la encuesta se propuso una estrategia y se elaboró un plan de acciones para el mejoramiento de la gestión en la instalación.

Palabras clave: Habilidades Directivas, Diagnóstico de Habilidades, Estrategia.

\section{Introducción.}

El éxito de una empresa depende de varios factores y del trabajo colectivo de todos sus integrantes. Es muy común escuchar que el trabajo en equipo es una pieza esencial dentro del perfecto engranaje que debe armarse para alcanzar las metas trazadas; y para que se hable de equipo tiene que existir obligatoriamente alguien que lo guíe y dirija.

Ese es precisamente el papel que le corresponden a los directivos quienes tienen la tarea de, con eficacia y eficiencia, conducir a su equipo de trabajo hacia resultados satisfactorios para la empresa. Queda claro entonces que semejante misión no pueda ser cumplida por cualquier persona, puesto que se requieren de habilidades y destrezas especiales para este tipo de desempeño.

Estas habilidades de los directivos son claves para el desarrollo de la empresa, por tanto, es menester de cada institución preocuparse porque cada uno de sus directivos las emplee en pos de mejorar el trabajo de su equipo.

Desgraciadamente, en el ámbito empresarial Cuba, no se manejan adecuadamente estas habilidades y en algunos casos ni siquiera se tienen en cuenta a la hora de establecer a un trabajador en un cargo directivo. Esto ha provocado que existan pocas investigaciones sobre esta temática aplicadas en las empresas cubanas, pese a la amplia bibliografía internacional existente referente al tema. 
El restaurante Don Cangrejo, perteneciente al grupo extrahotelero Palmares y entidad objeto de estudio de la presente investigación, pese a presentar buenos indicadores económicos, no se encuentra exento de esta problemática. Surgiría entonces una interrogante: ¿Poseen y hacen un buen uso de sus habilidades los directivos de la entidad para lograr una mejor gestión de la instalación?

La bibliografía acerca del desarrollo de las habilidades directivas, es amplia y variada, por tanto, los criterios son variables también. A lo largo de la historia cada autor ha dado su opinión acerca de la definición de las habilidades directivas, la cual han manifestado generalmente (según las fuentes consultadas por el colectivo de autores) determinando las diferentes habilidades que deben poseer, y en la mayoría de los casos no reparan en definir primero a ciencia cierta en que son en esencia las habilidades.

Pese a esto se encontraron 3 criterios que evidencian la evolución que ha tenido el concepto de habilidades directivas en los tiempos actuales.

El primero lo plantea Hampton (2009), al definir las habilidades directivas como: “ ... la individualidad y las relaciones interpersonales que definen la dirección".

Este criterio, aunque no es erróneo, no engloba en su totalidad el significado de las habilidades que debe poseer un directivo ya que estas no se limitan netamente al ámbito de las relaciones personales e interpersonales del directivo.

Un criterio más completo lo dan Whetten y Cameron (2011): “ Las habilidades directivas son el vehículo mediante el cual la estrategia y la práctica de la administración, las herramientas y las técnicas, los atributos de la personalidad y el estilo trabajan para producir resultados eficaces dentro de las organizaciones. En otras palabras, las habilidades directivas son los bloques de construcción sobre los que descansa la administración efectiva".

Esta definición no es solo más amplia, sino que además resalta la importancia de las habilidades en la efectividad de la administración.

El criterio más actual encontrado resume a las habilidades como “ ... lo que debe saber hacer un directivo". (Codina, 2014).

A partir de estos criterios el colectivo de autores define a las habilidades directivas como el conjunto de habilidades a nivel personal e interpersonal que deben poseer los directivos para lograr una gestión eficaz y eficiente.

Las habilidades esenciales de los directivos.

Whetten y Cameron (2011) definen 10 habilidades esenciales, las cuales dividen en 3 grupos: 
Habilidades personales.

- Desarrollo del autoconocimiento.

- Manejo del estrés personal.

- Solución analítica y creativa de problemas.

Habilidades interpersonales.

- Establecimiento de relaciones mediante una comunicación de apoyo

- Ganar poder e influencia.

- Motivación de los demás.

- Manejo de conflictos.

Habilidades grupales.

- Delegación.

- Formación de equipos efectivos y trabajo en equipo.

- Liderar el cambio positivo.

Esta división por grupos permite visualizar los planos donde actúan estas habilidades lo cual hace que esta categorización sea más organizada. Cave también resaltar que el mayor número de habilidades se encuentran en el grupo de las habilidades interpersonales en las que se evidencia que, contrario a lo que normalmente se cree, la dimensión interpersonal va más allá que la simple comunicación entre personas.

Respecto a este grupo de habilidades la Regional School of Public Administration of Montenegro, en su programa provisional para el curso “'Effective People Management Interpersonal Skills for Managers” el cual se efectuó del 23 al 25 de abril del 2013 planteó lo siguiente:

Las habilidades interpersonales no sólo incluyen cómo nos comunicamos con otros, incluye nuestra confianza y nuestra habilidad de escuchar y entender ".

Queda claro entonces que las relaciones interpersonales van más allá que el simple acto comunicativo.

Otro criterio sobre las habilidades fundamentales es el de Codina (2014) quien también destaca 10 habilidades como las principales que deben tener los cuadros. Estas son: 
1. Dirección o enfoque estratégico.

2. Comunicaciones interpersonales.

3. Estrategias y técnicas de negociación.

4. Manejo de conflictos.

5. Trabajo en equipo.

6. Liderazgo y motivación.

7. Diagnóstico de problemas y toma de decisiones.

8. Administración del tiempo y delegación.

9. Reuniones productivas.

10. Gerencia del cambio.

En sentido general, ambos criterios son semejantes, pero tienen también diferencias en cuanto a los nombres de cada una de las habilidades, los cuales están en correspondencia la propia visión que tienen cada uno de los autores de lo que son las habilidades directivas. Es preciso destacar la inclusión del liderazgo como una habilidad fundamental, ya que en ocasiones se tiende a equiparar al líder con el mánager, y estos no son iguales ya que un mánager puede ser un líder, pero un líder no precisamente tiene que ser un mánager.

Precisamente "el liderazgo atrae a diferentes tipos de hombres, entre ellos directivos, porque surge cuando una persona conduce y estimula al resto, para alcanzar un objetivo común". (Ayuso, 2013).

Por tanto “el papel del líder comprende más que determinar la estrategia simplemente. Esto incluye la clarificación de la dirección de la compañía, y de los procesos y políticas que motivan a los empleados y ayudan a que ellos logren sus objetivos comerciales". (Hrehová y Kamenec, 2011).

Entonces se puede afirmar que liderazgo y dirección son términos distintos, pero ambos son importantes dentro de una empresa. Sobre la relación entre estos términos Kumar y Jain (2013) plantean que la dirección y el liderazgo deben ir de la mano. Ellos no son la misma cosa, pero se unen necesariamente y se complementan. El trabajo del gerente es planear, organiza y coordina mientras que el trabajo del líder es inspirar y motivar.

Sobre la importancia de los líderes en las empresas Breckend (s/a) plantea:

A pesar de que las organizaciones exitosas de hoy dependen de la activa implicación de todos los empleados, los líderes son todavía a fin de cuentas responsables de establecer 
direcciones organizacionales a través de la visión y la estrategia. Cuando los líderes fracasan en el cumplimiento de esas responsabilidades, sus organizaciones pugnan. Cuando los líderes tienen éxito en el cumplimiento de esas responsabilidades, ellos pueden causar un gran impacto en el futuro de sus organizaciones ".

Apoyándose en los criterios anteriores, el colectivo de autores decidió establecer para la investigación las siguientes habilidades:

1. Desarrollo del autoconocimiento.

2. Manejo del estrés personal.

3. Solución analítica y creativa de problemas.

4. Comunicación interpersonal.

5. Motivación.

6. Flexibilidad.

7. Manejo de conflictos.

8. Delegación.

9. Formación y trabajo en equipo.

10. Liderazgo.

\section{Metodología.}

Para el grupo catalán ARCjob las habilidades son características personales estables y en buena parte inconscientes, que tienen un impacto positivo a largo plazo en el trabajo; se reflejan en comportamientos fácilmente identificables a través de la observación y/o entrevistas y, por tanto, pueden ser medidas. Y se pueden desarrollar mediante la experiencia y el aprendizaje.

Al ser medibles las habilidades es posible aplicar herramientas para su diagnóstico. Un ejemplo de herramienta lo constituye la rúbrica propuesta por Mejía (2015) en el Segundo Congreso Latinoamericano de Medición y Evaluación Educacional, la cual se propone para ser aplicada a los educadores de los centros escolares para evaluar el comportamiento de sus directores.

Otras Herramientas que se pueden aplicar según Hay Group (2007) son:

- Entrevistas de Incidentes Críticos (BEI). 
- Cuestionarios de Evaluación Multifuente.

La B.E.I. consiste en una entrevista altamente estructurada, profunda y detallada del desempeño pasado del candidato, la cual permite identificar y medir el grado de recurrencia, consistencia y solidez de las competencias del sujeto, evidenciadas en el repertorio de comportamientos que éste ha desplegado en su actuación exitosa como titular de un cargo en particular. Las investigaciones evidencian que, mientras más recurrentes y sólidas son las competencias del sujeto, mucho mejor y más exitoso es su desempeño profesional y gerencial.

Esta herramienta diseñada y utilizada ampliamente por el Dr. David Mc.Clelland y su equipo en Mc.Ber \& Company, sustenta los modelos de competencias bajo el supuesto básico de que, el mejor predictor del desempeño futuro de una persona es su desempeño pasado. (Quezada, s/a).

Los Cuestionarios de Evaluación Multifuente consisten en una herramienta (on-line o papel) que facilita la identificación de los niveles competenciales y/o de estilos de dirección de un colectivo, mediante la comparación entre la autoevaluación y la evaluación de los superiores y/o colaterales y/o colaboradores. (Hay Group, 2007).

En la presente investigación, se tomó como referencia el método de evaluación utilizado por el Hay Group en la BEI, según se muestra en la figura 1.

Figura 1. Herramienta utilizada por el Hay Group para el análisis de la BEI.

\begin{tabular}{|c|c|c|c|c|c|c|c|c|c|c|c|c|}
\hline \multicolumn{13}{|c|}{ ANÁLISIS COMPETENCIAL 2005} \\
\hline \multirow{2}{*}{ COMPETENCIAS } & \multicolumn{11}{|c|}{ DIRECTIVOS } & \multirow{2}{*}{$\begin{array}{c}\text { PERFIL } \\
\text { PROMEDIO }\end{array}$} \\
\hline & 1 & 2 & 3 & 4 & 5 & 6 & 7 & 8 & 9 & 10 & Total & \\
\hline FLEXIBILIDAD & 2 & 4 & 4 & 3 & 3 & 1 & 1 & 3 & 0 & & 21 & 2,33 \\
\hline IDENTIFICACIÓN CON LA ORGANIZACIÓN & 2 & 4 & 3 & 0 & 0 & 2 & 4 & 0 & 0 & & 15 & 1,67 \\
\hline PENSAMIENTO ANALITICO & 3 & 4 & 3 & 3 & 3 & 2 & 3 & 3 & 3 & & 27 & 3,00 \\
\hline PENSAMIENTO CONCEPTUAL & 2 & 3 & 3 & 2 & 2 & 0 & 2 & 2 & 2 & & 18 & 2,00 \\
\hline ORIENTACIÓN A RESULTADOS & 4 & 4 & 3 & 3 & 3 & 2 & 3 & 3 & 3 & & 28 & 3,11 \\
\hline INICIATIVA & 3 & 5 & 3 & 4 & 4 & 2 & 4 & 2 & 2 & & 29 & 3,22 \\
\hline ORIENTACIÓN AL CIUDADANO & 3 & 0 & 0 & 3 & 0 & 3 & 2 & 0 & 0 & & 11 & 1,22 \\
\hline COMPRENSIÓN DE LA ORGANIZACIÓN & 3 & 5 & 4 & 3 & 4 & 2 & 3 & 2 & 3 & & 29 & 3,22 \\
\hline IMPACTO E INFLUENCIA & 4 & 5 & 4 & 3 & 4 & 3 & 3 & 2 & 4 & & 32 & 3,56 \\
\hline DESARROLLO DE PERSONAS & 3 & 4 & 3 & 0 & 0 & 2 & 0 & 0 & 0 & & 12 & 1,33 \\
\hline LIDERAZGO & 2 & 4 & 3 & 3 & 4 & 2 & 2 & 2 & 3 & & 25 & 2,78 \\
\hline TRABAJO EN EQUIPO & 3 & 0 & 0 & 0 & 0 & 0 & 0 & 3 & 3 & & 9 & 1,00 \\
\hline
\end{tabular}

Fuente:http://www.insdapa.net/documents/análisis_competencias_andalucia/v0 1.0

Para realizar el diagnóstico, se tomó como referencia el método de evaluación utilizado por el Hay Group en la BEI (2017), pero fue adaptado a las particularidades de la 
investigación, realizándose un cuestionario en vez de una entrevista. El análisis de los resultados de la encuesta, aunque se realizó siguiendo el modelo empleado por Hay Group, también fue modificado por el colectivo de autores de esta investigación.

Para la aplicación de la herramienta se empleó la metodología siguiente:

1. Definición de la Población y la Muestra a estudiar.

2. Aplicación de la encuesta.

3. Tabulación de los datos.

4. Construcción de la Matriz de Análisis Competencial.

5. Análisis de los resultados.

6. Diagnóstico.

\section{Resultados.}

Población y Muestra: La instalación cuenta con una plantilla de 30 trabajadores, de los cuales diez son gastronómicos y seis trabajan en cocina; todos los trabajadores son profesionales con título de FORMATUR. La edad promedio es de 31 años, todos con más de 10 años de experiencia con buena estabilidad laboral.

Según el organigrama de la empresa, 11 de los 30 trabajadores, desempeñan de alguna manera u otra, funciones como directivos, aunque la mayoría no tengan un nivel jerárquico elevado. Estos trabajadores fueron tomados como población, y de ellos se escogió una muestra de 10, lo cual representa un $91 \%$ aproximadamente de la población, por lo que es una muestra bastante significativa.

Del total de trabajadores de la muestra, 8 son hombres y 2 son mujeres, la edad promedio oscila entre los 38 y 39 años y los años de experiencia rondan entre 11 y 12 años como promedio.

Aplicación de la encuesta y tabulación de los datos.

La encuesta aplicada consta con un bloque temático de 16 preguntas cerradas las cuales permitieron evaluar el estado de las 10 competencias directivas seleccionadas por este colectivo de autores en cada uno de los encuestados. Con cada pregunta se pudo asignar un valor en una escala del 1 al 4 a cada habilidad para la posterior construcción de la Matriz de Análisis Competencial. La escala para la medición del estado de las competencias se estableció de la siguiente manera:

1: Malo. 2: Regular. 3: Bueno. 4: Muy bueno. 
Para medir cada habilidad no se empleó más de 2 preguntas por cada una. En la siguiente tabla se muestra la relación de las habilidades con sus respectivas preguntas en el cuestionario.

Figura 2. Tabla de relación entre las preguntas y las habilidades evaluadas.

\begin{tabular}{|l|c|}
\hline \multicolumn{1}{|c|}{ Habilidades } & Pregunta (s) \\
\hline Desarrollo del autoconocimiento. & 1 y 2 \\
\hline Solución analítica y creativa de problemas. & 3 y 4 \\
\hline Manejo del estrés personal. & 5 y 6 \\
\hline Comunicación interpersonal. & 7 \\
\hline Motivación. & 8 \\
\hline Flexibilidad. & 9 \\
\hline Manejo de conflictos. & 10 y 11 \\
\hline Delegación. & 11 y 12 \\
\hline Formación y trabajo en equipo. & 13 y14 \\
\hline Liderazgo. & 15 y 16 \\
\hline
\end{tabular}

Fuente: Elaboración propia.

Tras la aplicación de la encuesta se agruparon los datos obtenidos en la siguiente tabla la cual muestra una relación porcentual entre las preguntas y la evaluación obtenida por cada encuestado:

Figura 3. Resultado de las encuestas.

\begin{tabular}{|c|c|c|c|c|}
\hline Preguntas & \multicolumn{4}{|c|}{ Puntuación en la escala } \\
\hline & 4 & 3 & 2 & 1 \\
\hline 1 & 70 & 30 & 0 & 0 \\
\hline 2 & 80 & 20 & 0 & 0 \\
\hline 3 & 10 & 30 & 60 & 0 \\
\hline 4 & 60 & 40 & 0 & 0 \\
\hline 5 & 90 & 10 & 0 & 0 \\
\hline 6 & 80 & 20 & 0 & 0 \\
\hline 7 & 10 & 0 & 0 & 0 \\
\hline 8 & 80 & 20 & 0 & 0 \\
\hline 9 & 80 & 20 & 0 & 0 \\
\hline 10 & 10 & 0 & 0 & 0 \\
\hline 11 & 0 & 20 & 30 & 50 \\
\hline 12 & 0 & 10 & 30 & 60 \\
\hline
\end{tabular}




\begin{tabular}{|c|c|c|c|c|}
13 & 60 & 30 & 10 & 0 \\
\hline 14 & 40 & 40 & 20 & 0 \\
\hline 15 & 30 & 40 & 20 & 10 \\
\hline 16 & 60 & 20 & 20 & 0 \\
\hline
\end{tabular}

Fuente: Elaboración propia.

\section{Construcción de la Matriz de Análisis Competencial.}

Utilizando el método de análisis planteado por el Hay Group, tras la aplicación de la encuesta antes mencionada se confeccionó la siguiente tabla la cual se nombró como "Matriz de Análisis Competencial", debido a que a pesar de guardar semejanza con la del Hay Group, es una matriz completamente nueva y arroja resultados diferentes. Dotada de un menor nivel de profundidad y facilidad de aplicación en PYMES y entidades similares.

Figura 4. Matriz de Análisis Competencial.

\begin{tabular}{|c|c|c|c|c|c|c|c|c|c|c|c|c|}
\hline \multicolumn{13}{|l|}{ Análisis Competencial } \\
\hline \multirow{2}{*}{ Competencias } & \multicolumn{11}{|c|}{ Directivos } & \multirow[b]{2}{*}{ Perfil Promedic } \\
\hline & 1 & 2 & 3 & 4 & 5 & 6 & 7 & 8 & 9 & 10 & Total & \\
\hline \multirow{2}{*}{ Desarrollo del autoconocimiento } & 4 & 4 & 4 & 3 & 4 & 4 & 3 & 3 & 4 & 4 & 37 & 3,7 \\
\hline & 3 & 4 & 4 & 4 & 4 & 4 & 4 & 3 & 4 & 4 & 38 & 3,8 \\
\hline \multirow{2}{*}{ Manejo del estrés personal } & 3 & 2 & 2 & 2 & 4 & 3 & 2 & 2 & 3 & 2 & 25 & 2,5 \\
\hline & 4 & 3 & 3 & 4 & 4 & 4 & 4 & 3 & 4 & 3 & 36 & 3,6 \\
\hline \multirow{2}{*}{ Solución analítica y creativa de problemas } & 4 & 4 & 4 & 4 & 4 & 4 & 4 & 3 & 4 & 4 & 39 & 3,9 \\
\hline & 3 & 4 & 4 & 4 & 4 & 4 & 4 & 3 & 4 & 4 & 38 & 3,8 \\
\hline Establecimiento de relaciones & 4 & 4 & 4 & 4 & 4 & 4 & 4 & 4 & 4 & 4 & 40 & 4 \\
\hline Motivación de los demás & 4 & 4 & 4 & 4 & 3 & 4 & 4 & 4 & 3 & 4 & 38 & 3,8 \\
\hline Flexibilidad & 4 & 4 & 3 & 4 & 4 & 3 & 4 & 4 & 4 & 4 & 38 & 3,8 \\
\hline Manejo de Conflictos & 4 & 4 & 4 & 4 & 4 & 4 & 4 & 4 & 4 & 4 & 40 & 4 \\
\hline \multirow{2}{*}{ Eacultamiente y delegación } & 1 & 3 & 1 & 2 & 2 & 1 & 3 & 2 & 1 & 1 & 17 & 1,7 \\
\hline & 2 & 1 & 1 & 1 & 1 & 1 & 2 & 2 & 3 & 1 & 15 & 1,5 \\
\hline \multirow{2}{*}{ Formación de equipos efectivos y trabajo en equipo } & 4 & 4 & 4 & 2 & 3 & 4 & 4 & 3 & 4 & 3 & 35 & 3,5 \\
\hline & 3 & 3 & 4 & 4 & 4 & 2 & 3 & 4 & 3 & 2 & 32 & 3,2 \\
\hline \multirow{2}{*}{ Liderazgo } & 2 & 3 & 2 & 1 & 3 & 4 & 4 & 3 & 4 & 3 & 29 & 2,9 \\
\hline & 4 & 4 & 4 & 4 & 2 & 3 & 4 & \begin{tabular}{|l|l|}
4 \\
\end{tabular} & \begin{tabular}{|l|l|}
3 \\
\end{tabular} & 2 & 34 & 3,4 \\
\hline
\end{tabular}

Fuente: Elaboración propia.

Análisis de los resultados y Diagnóstico.

La matriz arroja como resultados, que la entidad puede considerar como principales puntos fuertes de los directivos el establecimiento de relaciones, el manejo de conflictos y la solución analítica y creativa de problemas. En contraste con el manejo del estrés personal, el liderazgo y la delegación, que se encuentran en el área de mejora. Pese a estas 
deficiencias el colectivo de autores considera que de manera general los directivos de la entidad poseen las habilidades necesarias para una correcta gestión de la instalación. Este diagnóstico es respaldado por los indicadores económicos satisfactorios que presenta la instalación.

De otra forma y comparando con el perfil meta o perfil de excelencia se pueden observar las siguientes diferencias la cuales se recogieron en una tabla:

Figura 5. Tabla de diferencias entre el perfil meta y el perfil actual.

\begin{tabular}{|l|l|l|l|l|}
\hline \multirow{2}{*}{ No. } & \multirow{2}{*}{ Competencias } & Perfil de Excelencia o meta & Perfil Actual & Diferencia \\
\hline \multirow{2}{*}{1} & \multirow{2}{*}{$\begin{array}{l}\text { Desarrollo del } \\
\text { autoconocimiento }\end{array}$} & 4 & & \\
\hline \multirow{2}{*}{2} & \multirow{2}{*}{ Manejo del estrés personal } & 4 & 3,7 & 0,3 \\
\hline \multirow{2}{*}{3} & \multirow{2}{*}{$\begin{array}{l}\text { Solución analitica y creativa de } \\
\text { problemas }\end{array}$} & 4 & 3,8 & 0,2 \\
\hline 4 & Establecimiento de relaciones & 4 & 2,5 & 1,5 \\
\hline 5 & Motivación de los demás & 4 & 3,6 & 0,4 \\
\hline 6 & Flexibilidad & 4 & 3,9 & 0,1 \\
\hline 7 & Manejo de Conflictos & 4 & 3,8 & 0,2 \\
\hline \multirow{2}{*}{8} & \multirow{2}{*}{ Delegación } & 4 & 4 & 0 \\
\hline \multirow{2}{*}{9} & Formación de equipos efectivos & 4 & 3,8 & 0,2 \\
\hline & y trabajo en equipo & 4 & 3,8 & 0,2 \\
\hline \multirow{2}{*}{10} & \multirow{2}{*}{ Liderazgo } & 4 & 4 & 0 \\
\hline & & 4 & 1,7 & 2,3 \\
\hline
\end{tabular}

Fuente: Elaboración propia.

Figura 6. Diferencias entre el perfil meta y el perfil actual.

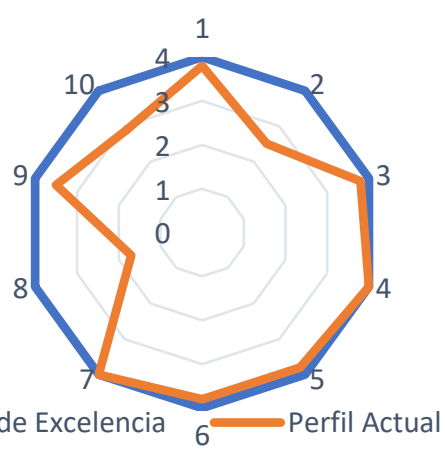

Fuente: Elaboración propia. 
Estos gráficos demuestran con claridad cuales habilidades presentan problemas y cuales son puntos fuertes. De acuerdo con el diagnóstico realizado en esta investigación se recomienda lo siguiente para mejorar las habilidades más débiles de sus directivos:

\section{Liderazgo:}

- Es necesario desarrollar un Plan de Formación específica, con herramientas concretas para poder gestionar equipos.

- Los directivos deben adecuar sus estilos de dirección a las personas, y no al grupo en general. Para ello, deben recibir formación en habilidades, pero sobre todo seguimiento y ayuda a la hora de implantar planes de acción con los colaboradores, para que dichos planes sean objetivos, alcanzables y realistas.

- Se debería programar una evaluación periódica del nivel directivo que evalúe la correcta aplicación de las funciones directivas. Para implantar una evaluación objetiva y adecuada a las necesidades de la función pública, se debería diseñar un Sistema de Gestión del Desempeño para los directivos de la Junta: dicho sistema facilitaría la comunicación con los niveles superiores y mejoraría la fijación de objetivos.

- Establecer con una periodicidad mensual, una reunión de trabajo operativa con todos los integrantes del servicio, con un orden del día comunicado con antelación por el Jefe de Servicio, y donde se tratarán los objetivos y temas a desarrollar en un determinado plazo temporal. Las reuniones deben ayudar a priorizar las acciones, diseñar estrategias, repartir el trabajo en función de los conocimientos/experiencia, facilitar la comunicación y la aportación de ideas, mejorando la relación interpersonal.

- En el mismo sentido, deberían establecerse unos talleres de trabajo para el desarrollo de las competencias directivas, donde los Jefes de Servicio que lideran las reuniones de sus equipos, puedan compartir e intercambiar impresiones que faciliten la mejora en la calidad directiva.

\section{Manejo del estrés personal:}

- Es necesario establecer un sistema de gestión de prioridades a nivel de la organización y su comunicación a cada uno de los directivos para lograr de forma planificada el cumplimento en tiempo de los objetivos. Este debe ser aprobado y establecido por la máxima autoridad y orientados según la misión y la visión de la entidad.

- Sería bueno aprovechar las fortalezas del trabajo en equipo para ver los problemas como oportunidades para mejorar, y no como limitaciones e inconvenientes. 
- La periodicidad y actualización de las prioridades evita el agrupamiento excesivo del trabajo a realizar a corto plazo y por lo tanto genera facilidades del estrés en el personal. Un control adecuado por parte de los directivos cubre con eficiencia este aspecto.

\section{Delegación:}

- A pesar de presentar una estructura organizacional relativamente simple, la capacidad de delegar funciones no puede ser pasada por alto. En caso de ausencia de un directivo superior el proceso de toma de decisiones debe realizarse de manera congruente, para ello debe existir una reserva de cuadros con amplio conocimiento de sus funciones, las que debe adquirir con la práctica de las responsabilidades delegadas en sí.

- Los directivos no pueden llevar adelante la empresa por si solos, por elevada que sea su eficiencia y efectividad sin delegar autoridad su trabajo es impreciso. Se sugiere reforzar la organización del restaurante, y de ser posible simplificar la dualidad existente en algunos puestos de trabajo para el logro de un enfoque más preciso hacia los objetivos de la organización.

Para mejorar en estos aspectos la instalación deberá adoptar la siguiente estrategia:

Garantizar una gestión eficaz de la instalación de la entidad aprovechando y potenciando las habilidades de los directivos que constituyen sus puntos fuertes y mejorando las que tienen afectadas mediante una capacitación eficaz y una evaluación continua de su desempeño, de forma tal que la entidad pueda detectar las principales deficiencias de sus cuadros para corregirlas; logrando así un cambio positivo constante en la empresa.

Teniendo en cuenta las recomendaciones hechas y la estrategia propuesta se elaboró el siguiente plan de acciones para la entidad: 
Figura 7. Plan de Acciones de mejora.

\begin{tabular}{|c|c|c|c|c|c|c|}
\hline $\mathrm{No}$ & Actividad & Procedimiento & Responsablo & Ejecutor & Tiempo & Actividad de Contingencis \\
\hline 1 & $\begin{array}{l}\text { Llevar a cabo cursos de } \\
\text { capacitación especifica } \\
\text { para cada uno de los } \\
\text { cuadros. }\end{array}$ & $\begin{array}{l}\text { Identificar los aspectos de mejora } \\
\text { en cada uno de los cuadros } \\
\text { Asignar a cada directivo un curso } \\
\text { especifico en dependencia de sus } \\
\text { debilidades }\end{array}$ & Administrador & Jefe de RRHH & Anual & $\begin{array}{l}\text { Asignar cursos alternativos } \\
\text { a cada directivo para } \\
\text { mejorar las habilidades que } \\
\text { lo requieran }\end{array}$ \\
\hline 2 & $\begin{array}{l}\text { Diseñar un Sistema de } \\
\text { Gestion del Desempeho } \\
\text { para los directivos }\end{array}$ & $\begin{array}{l}\text { Realizar reunión de directivos para } \\
\text { determinar los aspectos a tener en } \\
\text { cuenta en el sistema a diseh̆ar }\end{array}$ & Jefe de RRHH & Jefe de RRHH & Trimestral & $\begin{array}{l}\text { Diseñar un nuevo sistema } \\
\text { con otros aspectos a evaluar }\end{array}$ \\
\hline 3 & $\begin{array}{l}\text { Realizar evaluaciones } \\
\text { periodicas a nivel de } \\
\text { cuadros }\end{array}$ & $\begin{array}{l}\text { Llevar control del desempeho de } \\
\text { los directivos } \\
\text { Realizar reunión para llevar a cabo } \\
\text { la evaluación del desempeho }\end{array}$ & Administrador & Jefe de RRHH & Semestral & $\begin{array}{l}\text { Reestructurar el mecanismo } \\
\text { de las evaluaciones }\end{array}$ \\
\hline 4 & $\begin{array}{l}\text { Realizar reuniones de } \\
\text { trabajo operativas con } \\
\text { todos los integrantes del } \\
\text { servicio }\end{array}$ & $\begin{array}{l}\text { Informar al personal } \\
\text { Elaborar orden del dia } \\
\text { Discutir los objetivos a cumplir } \\
\text { para el plazo de tiempo } \\
\text { determinado }\end{array}$ & Administrador & Administrador & Mensual & $\begin{array}{l}\text { Redisehar los objetivos y } \\
\text { tareas a cumplir en el plazo } \\
\text { de tiempo }\end{array}$ \\
\hline 5 & $\begin{array}{l}\text { Realizar talleres de } \\
\text { trabajo para el desarrollo } \\
\text { de las competencias } \\
\text { directivas }\end{array}$ & $\begin{array}{l}\text { Determinar los lideres o jefes de } \\
\text { cada taller de trabajo } \\
\text { Llevar a cabo reuniones donde } \\
\text { compartan sus experiencias } \\
\text { intercambien experiencias }\end{array}$ & Jefe de RRHH & Jefe de RRHH & Semanal & $\begin{array}{l}\text { Realizar talleres de manera } \\
\text { más novedosa e } \\
\text { incentivadora ylo determinar } \\
\text { nuevos jefes de talleres }\end{array}$ \\
\hline 6 & $\begin{array}{l}\text { Establecer un sistema } \\
\text { de gestión de } \\
\text { prioridades a nivel de la } \\
\text { organización }\end{array}$ & $\begin{array}{l}\text { Ulevar a cabo reunión a nivel de } \\
\text { directivos para establecer el orden } \\
\text { de prioridades en la organización }\end{array}$ & Administrador & Administrador & Mensual & $\begin{array}{l}\text { Reestablecer el sistema de } \\
\text { gestion con otras } \\
\text { prioridades en la } \\
\text { organización }\end{array}$ \\
\hline 7 & $\begin{array}{l}\text { Llevar a cabo cursos de } \\
\text { capacitación para las } \\
\text { reservas de cuadros }\end{array}$ & $\begin{array}{l}\text { Identificar el posible puesto a } \\
\text { ocupar por cada reserva } \\
\text { Determinar las dificultades a } \\
\text { trabajar en cada reserva Asignar } \\
\text { a cada reserva un determinado } \\
\text { curso en dependencia de las } \\
\text { debilidades que presenta }\end{array}$ & Jefe de RRHH & Jefe de RRHH & Semanal & $\begin{array}{l}\text { Asignar cursos alternativos } \\
\text { a cada una de las reservas } \\
\text { en dependencia de las } \\
\text { habilidades que necesiten } \\
\text { ser mejoradas en cada uno }\end{array}$ \\
\hline
\end{tabular}

Fuente: Elaboración propia.

\section{Conclusiones:}

En la presente investigación se arribaron a las siguientes conclusiones:

- Las habilidades de los directivos ocupan un papel fundamental dentro de la gestión empresarial y son de vital importancia para el desarrollo de la empresa.

- Pese a las deficiencias detectadas tras el diagnóstico de las habilidades directivas de los cuadros del restaurante Don Cangrejo se comprobó que en sentido general estos poseen y hacen un buen uso de las habilidades.

- A pesar de presentar resultados satisfactorios, la entidad debe tomar acciones e implementar una estrategia para garantizar una mejor gestión a partir de las principales habilidades de sus directivos. 


\section{Referencias bibliográficas:}

ARCjob, Conoce y Practica la Entrevista de Incidentes Críticos. España. Disponible en: http://www.arcjob.net .Consultado el: 28/10/17. (s/a).

Ayuso, B., El liderazgo en la empresa. Trabajo de Fin de Grado. Escuela de Ciencias Empresariales Y del Trabajo de Soria. Universidad de Valladolid. España. 2013.

Codina, A., Habilidades Directivas. Editorial Academia. La Habana. Cuba. 17- 68. 2014

González, M. y Guenaga, G., Mecanismos de Influencia en las Organizaciones y Tácticas de Liderazgo. España. Disponible http://www.ehu.com/upv/mec_anismos_de_influen_cia. Consultado el: 4/12/17. (s/a).

Hay Group, Análisis de la situación actual de las competencias de las personas directivas de la administración de la Junta de Andalucía. Disponible en: http://www.insdapa.net/documents/análisis_competencias_andalucia/v01.00. Consultado el: 20/10/17. ( 2007).

Mejía, E., Evaluación de las Competencias Directivas. 2do Congreso Latinoamericano de Medición y Evaluación Educativa. México. (2013).

Quezada, H., La técnica de incidentes críticos-entrevista. Disponible en: http://www.gestiopolis.com/canales/derrhh/articulos/64/cltic.htm. Consultado el: 20/10/17. (s/a).

Ronda, G., Dirección Estratégica. Construcción y Dimensiones. Ediciones Futuro. La Habana. Cuba. 65-67. 2007.

Boston Consulting Group, The Managers of the 21st Century. 2020 Vision. Disponible en: https://www.bcg.com/documents/file14724.pdf. Consultado el: 13/10/17. (2006).

Brecken, D., Leadership Vision and strategic direction. Disponible en : The Quality Management Forum: www.asq1001.org . Consultado el : 8/12/17. (n/d).

Hopkins, T., Ten Essential Leadership Skills for Managers. Disponible en: www.hopkinsassociates.com/app/download/.../Ten+Essential+Skills+for+Manag ers.p. Consultado el: 20/10/17. (2009).

Hrehová, D. and Kamenec, P., The Management and Managerial Skills are needed for the Slovak Manage. Disponible en: http://www.aos.sk/casopisy/science/dokumenty/archiv/2011_2/10.pdf. Consultado el: 20/10/17. (2011).

Kumar, M. and Jain, S., Leadership Management: Principles, Models and Theories. Global Journal of Management and Business Studies. Volume 3, Number 3 (2013), pp. 309-318. . Disponible en: http://www.ripublication.com/gjmbs.htm.

Regional School of Public Administration of Montenegro, Effective People Management - Interpersonal Skills for Managers. Provisional Program.Disponible en: https://catalogue.pearsoned.co.uk/assets/hip/gb/hip_gb_pearsonhighered/sample chapter. Consultado el: 4/12/17. (2013). 


\section{PARA CITAR EL ARTÍCULO INDEXADO.}

Malvarez Cuello, A., Crispin Rodriguez, D., \& espinosa Gonzalez, C. A. (2020). Matriz de análisis competencial, herramienta para el desarrollo de habilidades directivas. Visionario Digital, 4(3), 32-47.

https://doi.org/10.33262/visionariodigital.v4i3.1271

\section{【 Ciencia}

El artículo que se publica es de exclusiva responsabilidad de los autores y no necesariamente reflejan el pensamiento de la Revista Visionario Digital.

El artículo queda en propiedad de la revista y, por tanto, su publicación parcial y/o total en otro medio tiene que ser autorizado por el director de la Revista Visionario Digital.
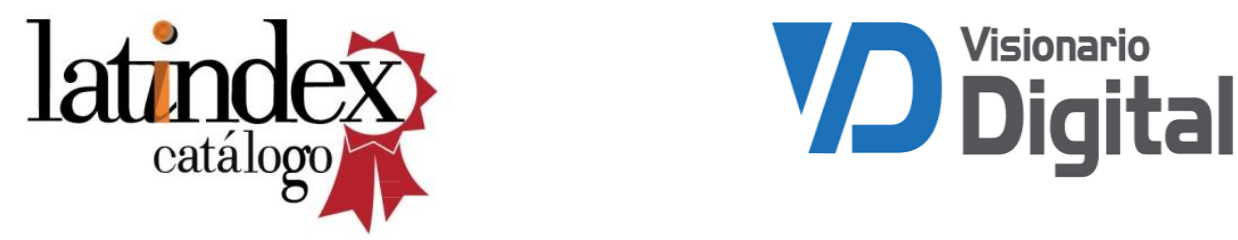